\title{
Presumptive Identification of Candida spp. from Clinical Isolates and Anticandidal Effect of Essential Oils
}

\author{
S. S. Khandare ${ }^{1}$, A.P. Moon ${ }^{2}$, M. G. Ingale ${ }^{3}$ \\ P.G.Department of Microbiology, Bajaj College of Science, Wardha. Maharashtra-442001. \\ Corresponding author email- ksuhas21@gmail.com
}

\begin{abstract}
Candida is normal flora of human skin, mouth, vagina, colon and causes mycosis worldwide. Candida causes oral thrush, vaginitis and other potentially life-threatening diseases. This study assesses anticandidal potential of essential oils against Candida species isolated from clinical samples obtained from Mahatma Gandhi Institute of Medical Sciences, Sewagram, Wardha, Maharashtra. On the basis of morphological characterization on Sabouraud dextrose agar, CHROMagar, Germ tube test and biochemical characterization, Candida species identified as Candida albicans, Candida krusei, Candida glabrata, Candida parapsilosis. Clove, thyme, cinnamon and eucalyptus essential oils with concentrations of 6.2, 12.5, 25, 50 and $100 \mu \mathrm{g} / \mathrm{ml}$ were used to study their anticandidal activity. C. albicans demonstrated zone of inhibition (ZOI) 29, 20, 32, 29 mm, Candida krusei 25, 25, 30, $21 \mathrm{~mm}$, Candida glabrata 24, 22, 31, 32mm,Candida parapsilosis 33,31,33, 29mm towards clove, thyme, cinnamon and eucalyptus oil (each at $100 \mu \mathrm{g} / \mathrm{ml}$ ) respectively which are highest ZOI as compared with antifungal agent Amphotericin-B, which showed 16, 13, 12, $8 \mathrm{~mm}$ against C. albicans, C. krusei, C.glabrata and C. parapsilosis respectively. All Candida spp. produced biofilms and enzyme lipase. This study will facilitate the development of novel broad-spectrum key molecules against a range of Candida species.
\end{abstract}

Key words: Candida spp., Essential oils, zone of inhibition, biofilm, lipase.

\section{Introduction:}

Candida is dimorphic fungus that exists as a commensal of warm-blooded animals including humans (Ashman et al., 1995). It is oval yeast with a single bud. It is a part of a normal flora of mucous membrane of the upper respiratory, gastrointestinal and female genital tracts (Levinson, 2008). Many changes in the internal and external environment induce this harmless saprophyte to become a true pathogen.

Fungi, once considered non-pathogenic microbiological curiosities have emerged as an important cause of community acquired and health care associated infections (Chakrabarti et al., 2000). Factors like HIV/AIDS, treatment with broad spectrum antibiotics and immunosuppressive drugs increase the vulnerability to fungal infections (Deorukhkar et al., 2012). Among various pathogenic species of fungi, Candida is the most prominent cause of fungal infections (Sullivan et al., 1996). Candida albicans is an opportunistic fungal pathogen that exists as a harmless commensal in the gastrointestinal and genitourinary tracts in about $70 \%$ of humans and about $75 \%$ of women suffer from Candida infection at least once in their lifetime (Ruhnke et al., 2002). Although a part of normal microbiota, Candida is capable of causing various clinical manifestations ranging from mucocutaneous overgrowth to disseminated infections like candidemia (Eggimann et al., 2003). Virulence factors like tissue adhesion, phenotypic switching, biofilm formation and production of extracellular hydrolytic enzymes play an important role in colonization and invasion of host tissues (Sachin et al., 2012). The genus Candida is composed of heterogeneous group of organisms and more than 17 different Candida spp. are implicated in human infections (Pfaller et al., 2007). Advances in medical practice have increased the frequency of fungal infections in hospitalized patients. Fungal pathogens, now account for almost $10 \%$ of all nosocomial blood stream infections (BSIs) (Warnock et al., 1996). Among various fungal pathogens, Candida spp. is the important cause of substantial 
morbidity and mortality in hospitalized patients. It is fast becoming a very important pathogen among critically ill hospitalized patients (Rajkumari et al., 2014).

Essential oils are natural products produced by aromatic plants and are mainly composed of terpenes and terpenoids (Nazzaro et al., 2013). Being lipophilic, these oils typically integrate into membrane structures causing increased cell permeability, leaching of intracellular components and inactivation of enzymes (Sikkematb et al., 1994). Essential oils can act against Candida by inhibiting ergosterol synthesis (Adil et al., 2014), altering cell wall morphology (de Castro et al., 2013), inhibiting enzymes involved in cell wall synthesis (Bang et al., 2000), changing cell membrane permeability (Rajkowska et al., 2016) and producing oxygen reactive species (Singh et al., 2016). Antimicrobial, anti-aseptic, anti-inflammation and anti-oxidant activity of essential oils, alone and in combination with commercial agents is well known (Santos et al., 2012). The aim of this study was therefore to investigate the antifungal potential of four commercial essential oils ie. clove, thyme, cinnamon and eucalyptus oil against Candida species. Antifungal activity of commercial essential oils was compared to Amphotericin-B, Terbinafine, Ketoconazole and Fluconazole. These findings highlight the in vitro efficacy of certain essential oils against Candida species.

\section{Methodology}

\section{Sample collection:}

For the isolation of Candida species, various clinical specimens like urine, sputum, endo-cervical and vaginal swabs (VS), urethra swabs, wound and eye swabs were collected from department of Microbiology, Mahatma Gandhi Institute of Medical Sciences, Sewagram, Wardha, Maharashtra. All the specimens were preserved until use according to the standard microbiological procedures (Jay et al., 2013).

\section{Procurement of essential oils}

Selected essential oils (Clove, Thyme, Cinnamon and Eucalyptus) were purchased from the local market of Wardha, Maharashtra. These oils were selected based on their use in traditional medicine.

\section{Isolation of Candida species from clinical samples}

For isolation of Candida species from clinical samples, Sabouraud's Dextrose Agar (SDA) supplemented with chloramphenicol $(0.05 \mathrm{mg} / \mathrm{ml})$ and cyclohexamide $(0.5 \mathrm{mg} / \mathrm{ml})$ was used. Samples were inoculated on the sabourads dextrose agar plates. Plates were incubated at $37^{\circ} \mathrm{C}$ at $24 \mathrm{hrs}$. White to cream-colored, pasty and smooth colonies appeared within 24 hours to $48 \mathrm{hrs}$. on SDA. The samples were sub cultured and were maintained on SDA slants at $4^{\circ} \mathrm{C}$. (Samyuktha et al., 2017).

\section{Identification of Candida species}

The colonies morphologically resembling to the members of genus Candida were subjected to Gram staining and Lactophenol cotton blue staining. Isolates were also characterized biochemically by performing indole test, tellurite reduction test, tetrazolium reduction test and also by performing germ tube test. Once the colonies were confirmed, speciation was done by inoculating Candida spp. on CHROMagar and incubated at $37^{\circ} \mathrm{C}$ for $24 \mathrm{hrs}$. The species were identified by morphology of the colonies on CHROMagar. CHROMagar candida is a novel, differential culture medium that is claimed to facilitate the isolation by colorimetric presumptive identification (Kashid et al., 2011 \& Vijaya et al., 2011). 


\section{Determination of virulence factors of Candida sp.}

The various virulence factors of candida species are determined as follows

\section{A) Determination of Phospholipase production}

The phospholipase activity of Candida species was determined by using egg yolk agar medium. Approximately $5 \mu 1$ of the inoculum of the Candida strain was aseptically inoculated onto the egg yolk agar. The plates were dried at room temperature and then incubated at $37^{\circ} \mathrm{C}$ for $48 \mathrm{~h}$. The plates were examined in terms of the presence of precipitation zone around the colony. The presence of precipitation zone indicates phospholipase enzyme production (Samaranayake et al., 1984).

\section{B) Determination of protease activity}

For determination of Protease activity of Candida species, a loopful of culture was introduced to a sterile filter paper disc and placed on the surface of bovine serum albumin agar (BSA; $\mathrm{pH}$ : 5.0). The inoculated plates were incubated at $37^{\circ} \mathrm{C}$ for two days, and the diameter of the inhibition zone around the discs was measured to determine protease activity (Gokce et al., 2007).

\section{C) Determination of Lipase activity}

A precipitation test using Tween 80 agar plates was carried out to confirm lipolytic activity. Tween 80 substrate plates were prepared as follows (g/L); $10 \mathrm{~g}$ peptone, $5 \mathrm{~g} \mathrm{NaCl}_{2}, 0.1 \mathrm{~g} \mathrm{CaCl}_{2} \cdot 2 \mathrm{H}_{2} \mathrm{O}, 20 \mathrm{~g}$ agar and $10 \mathrm{ml}(\mathrm{v} / \mathrm{v}$ ) Tween 80. This method is based on the principle of calcium salt precipitation. Due to action of lipase enzyme, hydrolysis of tween 80 releases fatty acids which bind with the calcium in the medium to form insoluble crystals around the point of inoculation. Tween 80 is used for the detection of lipases as it contains esters of oleic acid. The organisms were inoculated onto the plates and incubated at $37^{\circ} \mathrm{C}$ for $2-4$ days. A white precipitation around the boundary of the colony was indicative of lipase activity (D. Kumar et al., 2012).

\section{D) Determination of Biofilm production}

Biofilm formation was determined by visual method. In this method a loopful of colonies from the surface of Sabouraud Dextrose Agar (SDA) plate was inoculated into a polystyrene tube (Falcon conical tube with a screw cap), containing $10 \mathrm{ml}$ of Sabouraud Dextrose Broth (SDB), supplemented with glucose (final concentration of $8 \%$ ). After incubation at $35^{\circ} \mathrm{C}$ for $48 \mathrm{~h}$, broth in the tubes was gently aspirated. The tubes were washed with distilled water twice and then stained with safranin $2 \%$ for 10 min. Afterwards, they were tested to determine the presence of an adherent layer. With respect to adherence to the surface of the tube, biofilm production was scored as strong (+++), moderate (++) and weak (+). Similarly Biofilm production was also assessed on Tryptic soy broth $\&$ Congo red agar medium (Yigit et al., 2011).

\section{Evaluation of Anticandidal activity of essential oils by Agar well diffusion assay}

The anticandidal activities of the selected essential oils were determined by Agar well diffusion assay techniques (Reeves et al., 1989). In this method, $100 \mu \mathrm{l}$ of standardized inoculum of each test fungus were spread onto sterile Sabouraud Dextrose Agar. Essential oils with concentrations of 6.2, 12.5, 25, 50 and $100 \mu \mathrm{g} / \mathrm{ml}$ were used to study their anticandidal activity. Well with $8 \mathrm{~mm}$ diameter was cut from the agar using a sterile cork-borer and subsequently each well was filled with the appropriate concentrations of essential oils. The plates were kept at room temperature for $1 \mathrm{hr}$ to allow proper diffusion of the oil into agar and then incubated at $30{ }^{\circ} \mathrm{C}$ for $3-5$ days. Triplicates were prepared for each sample. The essential oils having antimicrobial activity inhibit the fungal growth and the clear zones were formed. The zone of inhibition was measured in millimeters (Raid et al., 2014). 


\section{Evaluation of Anticandidal activity of standard antifungal drugs}

For comparative analysis of sensitivity of Candida sp. to essential oils and antifungal drugs, a disc diffusion assay as recommended by Clinical and Laboratory Standards Institute (CLSI) was performed to determine the susceptibility of the test isolates to standard antifungal drugs. Briefly, $100 \mu \mathrm{l}$ of candida suspension adjusted to a 0.5 McFarland standard was streaked onto SDA plates and then discs of antifungal drugs (Amphotericin-B (AMB), Terbinafine (TRF), Ketoconazole (KTC), Fluconazole (FLC) $(100 \mu \mathrm{g} / \mathrm{disc}))$, were placed over the agar surface and incubated at $37^{\circ} \mathrm{C}$ for $24 \mathrm{~h}$. The diameter of the zone of inhibition around the discs was recorded in mm. Each experiment was conducted in triplicate and the average zone size was calculated (Clinical \& Laboratory standards Institute USA 2009).

\section{Results and Discussion:}

In the present study, four candida species were isolated from clinical samples obtained from Mahatma Gandhi Institute of Medical Sciences, Sewagram, Wardha. On the basis of morphological \& biochemical characteristics \& growth on differential culture medium CHROMagar these candida species were identified as Candida albicans, Candida krusei, Candida glabrata, Candida parapsilosis. All the four species were found to be gram positive \& also showed positive results for germ tube test, tellurite reduction test \& tetrazolium reduction test. Among the four isolates, Candida albicans and Candida parapsilosis were also found to produce indole. The speciation of isolates was done by studying their growth on CHROMagar. On this medium, Candida albicans, Candida krusei, Candida glabrata, Candida parapsilosis produce Green, purple and white colour colonies respectively (Table 1 \& Fig 1). Chromogenic agar is a newer and more rapid method to speciate candida, which contains enzymatic substrates that are linked to chromogenic compounds. When specific enzyme cleaves the substrate, chromogenic substances produce colour. The action of different enzymes produced by yeast species results in color variation which is useful for the presumptive identification of some yeasts (Odds et al., 1994). But we found that CHROMagar is an effective and faster screening method which can be used for speciation of Candida. In the present study CHROM agar could identify, Candida albicans, Candida tropicalis and Candida glabrata. Similar findings have been reported by different studies (Golia et al., 2013).

All the isolates were found to produce lipase enzyme as marker of their virulence (Table 2). As far as the biofilm formation is concern, all the isolates were found to form the biofilm. Candida albicans was found to produce strong $(+++)$, moderate $(++) \&$ strong $(+++)$ biofilm on Tryptic soy broth, Sabouraud Dextrose Broth (SDB) \& Congo red agar medium respectively. Candida krusei was found to produce moderate (++), weak (+) \& moderate (++) biofilm on Tryptic soy broth, SDB \& Congo Red agar medium respectively. Candida glabrata showed strong biofilm production on Tryptic soy broth and SDB, but no biofilm production was observed on Congo red medium. Percent biofilm production by Candida parapsilosis was found to be weak (+), strong (+++) and weak (+) on Tryptic soy broth, SDB \& Congo red agar medium respectively (Table 3).

Essentials oils have been tested for in vitro anticandidal activity and demonstrated to have potential anticandidal effect. Essential oils with concentrations of 6.2, 12.5, 25, 50 and $100 \mu \mathrm{g} / \mathrm{ml}$ were used to study their anticandidal activity. C. albicans demonstrated zone of inhibition (ZOI) 29, 20, 32, $29 \mathrm{~mm}$, Candida krusei 25, 25, 30, $21 \mathrm{~mm}$, Candida glabrata 24, 22, 31,32 mm, Candida parapsilosis 33, 31, 33, $29 \mathrm{~mm}$ towards clove, thyme, cinnamon and eucalyptus oil (each at $100 \mu \mathrm{g} / \mathrm{ml}$ ) respectively which are highest ZOI as compared with antifungal agent Amphotericin-B which showed 16, 13, 12, $8 \mathrm{~mm}$ against C. albicans, C. krusei, C.glabrata and $C$. parapsilosis respectively. Anticandidal activity shown by clove and cinnamon oils (ZOI $33 \mathrm{~mm}$ each) against Candida parapsilosis was found to be more than antifungal agent Terbinafine (ZOI of $31 \mathrm{~mm}$ ). Anticandidal activity of essential oils was also found to be more as compared to ketoconazole and fluconazole. Activity of clove and cinnamon oil was found to be more (29 and $32 \mathrm{~mm}$ each) against $C$. albicans as compared to fluconazole (28 
$\mathrm{mm}$ ). The highest zone of inhibition was found to be against Candida parapsilosis ie. $33 \mathrm{~mm}$ each shown by clove and cinnamon oil respectively. The highest zone of inhibition shown by clove, thyme and eucalyptus oil was found to be against $C$. parapsilosis $(33 \mathrm{~mm})$, . parapsilosis $(31 \mathrm{~mm})$, C. parapsilosis $(33 \mathrm{~mm})$, C. glabrata $(32 \mathrm{~mm})$ respectively. While the lowest activity was recorded against $C$. albicans for thyme oil (ZOI $20 \mathrm{~mm}$ ). Our result showed that cinnamon oil has higher anticandidal activity against all the tested candida species. The antimicrobial properties of Cinnamon are thought to be due to eugenol and cinnamaldehyde (Matan et al., 2006). Cinnamon bark has cinnamaldehyde as its major component, while cinnamon leaf oil has eugenol rather than cinnamaldehyde. Eugenol is a phenolic compound, and several authors have reported its high antimicrobial activity in phenolic structured compounds (Suhr et al., 2003). Many publications have reported about significant antimicrobial effects of cinnamon and clove essential oils against important fungal pathogens: These results was in conformity with the previous studies, which reported that clove, thyme and cinnamon essential oils exhibited an inhibitory effect against a wide range of pathogenic fungi. Radwan et al., 2014 tested antifungal activities of the essential oils against the fungi \& his findings revealed that thyme oil completely inhibited the growth of different fungal isolates at concentrations of $0.25,0.5$ and $1 \%$. Clove and cinnamon oils completely inhibited the growth of different fungal isolates at a concentration of $6 \%$.

Table 1: Morphological and Biochemical Characterization of Candida sp.

\begin{tabular}{|c|c|c|c|c|}
\hline Tests & C. albicans & C. krusei & C. glabrata & C.parapsilosis \\
\hline Grams staining & + & + & + & + \\
\hline Germ tube test & + & + & + & + \\
\hline Indole test & + & - & - & + \\
\hline Tellurite reduction & + & + & + & + \\
\hline Tetrazolium reduction & + & + & + & + \\
\hline Colonies on SDA & White & White & Cream & Cream \\
\hline CHROMagar & Green & Purple & White & White \\
\hline
\end{tabular}

Table 2: Studies on virulence markers

\begin{tabular}{|c|c|c|c|c|}
\hline Virulence factors & C. albicans & C. krusei & C.gabrata & C. parapsilosis \\
\hline Phospholipase & - & - & - & - \\
\hline Lipase & + & + & + & + \\
\hline Proteinase & - & - & - & - \\
\hline
\end{tabular}

Table 3: Biofilm formation

\begin{tabular}{|c|c|c|c|c|}
\hline Medium & C. albicans & C. krusei & C. glabrata & C. parapsilosis \\
\hline Tryptic soy broth & Strong (+++) & Moderate (++) & Strong (+++) & Weak $(+)$ \\
\hline SDA & Moderate (++) & Weak (+) & Strong (+++) & Strong $(+++)$ \\
\hline Congo red agar & Strong (+++) & Moderate (++) & - & Weak $(+)$ \\
\hline
\end{tabular}

Table 4: Anticandidal activity of essential oil \& antifungal agents

\begin{tabular}{|c|c|c|c|c|c|c|c|c|}
\hline \multirow{3}{*}{ Candida sp. } & \multicolumn{4}{|c|}{ Essential oils $(100 \mu \mathrm{g} / \mathrm{ml})$} & \multicolumn{4}{|c|}{$\begin{array}{l}\text { Antifungal agents } \\
(100 \mu \mathrm{g} / \text { disc })\end{array}$} \\
\hline & \multicolumn{4}{|c|}{ Zone of inhibition (in $\mathrm{mm}$ ) } & \multicolumn{4}{|c|}{ Zone of inhibition (in $\mathrm{mm}$ ) } \\
\hline & Clove & Thyme & Cinnamon & Eucalyptus & AMB & TRF & KTC & FLC \\
\hline C. albicans & $29 \pm 2$ & $20 \pm 1$ & $32 \pm 1$ & $29 \pm 1$ & $16 \pm 1$ & $32 \pm 1$ & $28 \pm 2$ & $28 \pm 2$ \\
\hline C. krusei & $25 \pm 2$ & $25 \pm 2$ & $30 \pm 2$ & $21 \pm 1$ & $13 \pm 2$ & $33 \pm 2$ & $29 \pm 1$ & $35 \pm 1$ \\
\hline C. glabrata & $24 \pm 2$ & $22 \pm 2$ & $31 \pm 1$ & $32 \pm 1$ & $12 \pm 2$ & $32 \pm 1$ & $28 \pm 2$ & $26 \pm 2$ \\
\hline $\begin{array}{c}C . \\
\text { parapsilosis }\end{array}$ & $33 \pm 1$ & $31 \pm 1$ & $33 \pm 1$ & $29 \pm 2$ & $08 \pm 1$ & $31 \pm 2$ & $27 \pm 2$ & $22 \pm 2$ \\
\hline
\end{tabular}


Fig 1: Growth of Candida species on CHROMagar

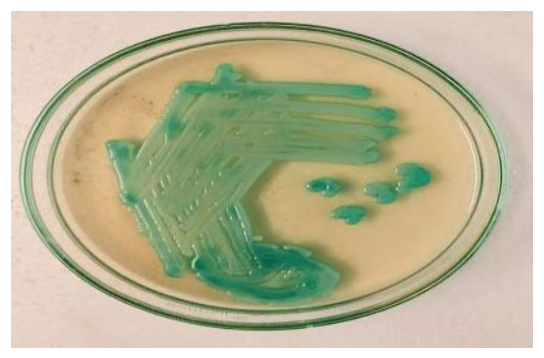

C. albicans

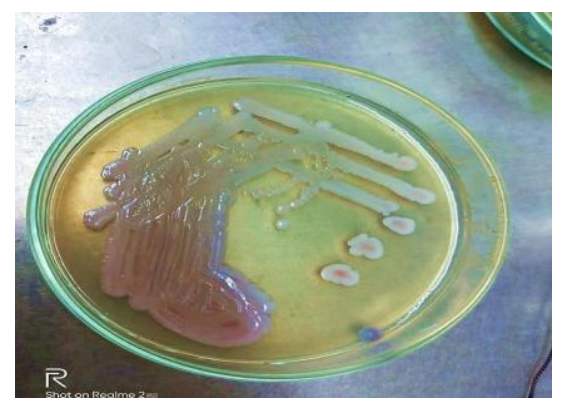

C. glabrata

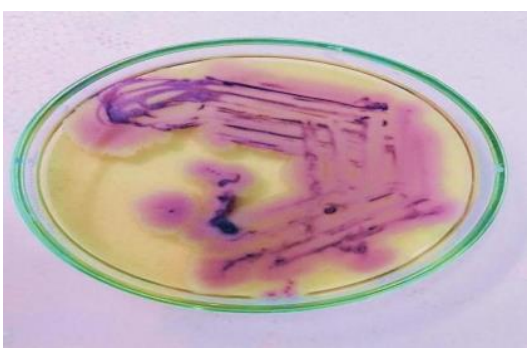

C. krusei

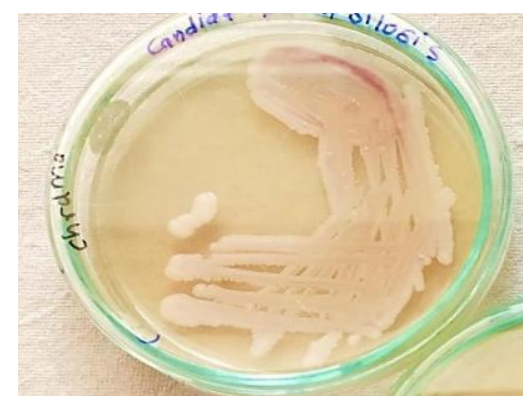

C. parapsilosis

\section{Conclusion:}

Essential oils like Clove, Thyme, Cinnamon \& Eucalyptus oils inhibited the growth of Candida species more efficiently than other antifungal agents. This comprehensive study will benefit researchers who wish to explore the potential of essential oils in the development of novel broad-spectrum key molecules against a broad range of Candida sp. Future scope of antifungal activity of essential oil will help to control Candida pathogens as well as diseases in humans.

\section{REFERENCES:}

1. Ashman RB, Fulurija A, Robertson TA, Papadimitriou JM. Rapid destruction of skeletal muscle fibers by micelial growth forms of Candida albicans. ExpMolPathol. 1995; 62(2):109-117. 2.

2. Levinson W. Medical Microbiology \& Immunology illustrated 10th edition. The McGraw-Hill Companies, 2008.

3. Chakrabarti, A., Kaur, R., and Das, S. 2000. Molecular methods in diagnosis of fungal infections. Indian J Med Microbiol.18:146-152.

4. Deorukhkar, S., Saini, S., and Jadhav, P. 2012 c. Evaluation of different media for germ tube production of Candida albicans and Candida dubliniensis. International Journal of Biomedical and Advance Research. 3:704-707.

5. Sullivan, D., Henman, M., Moran, G., et al. 1996. Molecular genetic approaches to identification, epidemiology and taxonomy of non-albicans Candida species. J Med Microbiol. 44:399-408. 
6. M. Ruhnke and G. Maschmeyer, "Management of mycoses in patients with hematologic disease and cancer-review of the literature," European Journal of Medical Research, vol. 7, no. 5, pp. 227-235, 2002.

7. Eggimann, P., Garbinm, J., and Pittet, D. 2003. Epidemiology of Candida species infections in critically ill non immunosuppressed patients. Lancet Infect Dis. 3: 685-702.

8. Sachin, C. D., Ruchi, K., and Santosh, S. 2012. In-vitro evaluation of proteinase, phospholipase and haemolysin activities of Candida species isolated from clinical specimens. Int J Med Biomed Res.1:153-1

9. Pfaller, M., and Diekema, D. 2007. Epidemiology of invasive candidiasis: A persistent public health problem. Clin Microbiol Rev. 20:133-163.57.

10. Warnock, D., and Cambell, C. 1996, Medical Mycology. Mycol Res. 100:1153-62.

11. Rajkumari, N., Mathur, P., Xess, I., and Misra, M. 2014. Distribution of different yeasts isolates among trauma patients and comparison of accuracy in identification of yeasts by automated method versus conventional methods for better use in low resource countries. Indian J. Med. Microbiol. 32: 391-94.

12. Nazzaro, F.; Fratianni, F.; De Martino, L.; Coppola, R.; De Feo, V. Effect of essential oils on pathogenic bacteria. Pharmaceuticals 2013, 6, 1451-1474.

13. Sikkematb, J.; de Bontt, J.A.M. Interactions of cyclic hydrocarbons with biological membranes. J. Biol. Chem. 1994, 269, 8022-8028. 13.

14. Adil, M.; Singh, K.; Verma, P.K.; Khan, A.U. Eugenol-induced suppression of biofilm-forming genes in Streptococcus mutans: An approach to inhibit biofilms. J. Glob. Antimicrob. Resist. 2014, 2, $286-292$.

15. De Castro, R.D.; Lima, E.O. Anti-Candida Activity and Chemical Composition of Cinnamomum zeylanicum Blume Essential Oil. Braz. Arch. Biol. Technol. 2013, 56, 749-755.

16. Bang, K.; Lee, D.; Park, H.; Rhee, Y. Inhibition of fungal cell wall synthesizing enzymes by transcinnamaldehyde. Biosci. Biotechnol. Biochem. 2000, 64, 1061-1063.

17. Rajkowska, K.; Nowak, A.; Kunicka-styczy, A.; Siadura, A. Biological effects of various chemically characterized essential oils: Investigation of the mode of action against Candida albicans and HeLa cells. RSC Adv. 2016, 6, 97199-97207.

18. Singh, S.; Fatima, Z.; Hameed, S. Citronellal-induced disruption of membrane homeostasis in Candida albicans and attenuation of its virulence attributes. Rev. Soc. Bras. Med. Trop. 2016, 49, 465-472.

19. Santos, K.K.; Matias, E.F.; Souza, C.E.; Tintino, S.R.; Braga, M.F.; Guedes, G.M.; Nogueira, L.F.; Morais, E.C.; Costa, J.G.; Menezes, I.R.; et al. Anti-candida activity of Mentha arvensis and Turnera ulmifolia. J. Med. Food 2012, 15, 322-324.

20. Jay S, Harita V. Candida Species Isolated from Various Clinical Samples and their Susceptibility Patterns to Antifungals. J Med Microbiol Infec Dis. 2013; 1(1):22-6.

21. Arasi Samyuktha A et al., Sch. J. App. Med. Sci., Aug 2017; 5(8F):3460-3468

22. Kashid $R$ A, Belawadii S, Devi G, Indumati. Characterization and antifungal susceptibility testing for candida species in a tertiary care hospital. Journal of Health Sciences and Research. August 2011; 2 (2): $1-7$.

23. Vijaya D., HarsHa T.r., NagaraTNamma T., Candida Speciation Using Chrom Agar The Journal of Clinical and Diagnostic Research. 2011 August. Vol-5(4): 755-758.

24. Samaranayake LP, Raeside JM, Macfarlane TW. Factors affecting the phospholipase activity of Candida species by invitro. Sabouraudia. 1984; 22(3):201-7.

25. Gokce G, Cerikcioglu N, Yagci A. Acid proteinase, phospholipase and biofilm production of Candida species isolated from blood cultures. Mycopathologia. 2007; 164(6):265-9.

26. D. Kumar, L. Kumar, S. Nagar, C. Raina, R. Parshad, V.K. Gupta. Screening, isolation and production of lipaselesterase producing Bacillus sp. strain DVL2 and its potential evaluation in esterification and resolution reactions. Arch. Appl. Sci. Res., 4 (2012), pp. 1763-1770.

27. Yigit N, Aktas E, Dagistan S, Ayyildiz A. Investigating biofilm production, Coagulase and Hemolytic activity in Candida species isolated from denture stomatitis patients. Eurasian J Med. 2011; 43(1):27-32. 
28. Reeves DS. Antibiotic assays. In: Hawkey PM, Lewis DA, editors. Medical bacteriology, a practical approach. Oxford: IRL Press; 1989. pp. 195-221.

29. Raid AA, Yazeed AS, Ayesha M, Rabbani SK, Janardhan C, Gupta VC. Evaluation of antibacterial activity of crude protein extracts from seeds of six different medical plants against standard bacterial strains. Saudi J Biol Sci. 2014;21:147-151. doi: 10.1016/j.sjbs.2013.09.003.

30. Clinical and Laboratory Standards Institute. Reference Method for Antifungal Disc Diffusion Susceptibility Testing of Yeasts. Proposed guideline M44-A2, Wayne, PA: Clinical and Laboratory Standards Institute USA, 2009.

31. Odds, F.C., Bernaerts, R. 1994. CHROM agar Candida, a new differential isolation medium for presumptive identification of clinically important Candida spp. J. Clin. Microbiol., 32(8): 1923-9.

32. Golia, S., Reddy, K.M., Karjigi, K.S., Hittinahalli, V. 2013. Speciation of Candida using chromogenic and cornmeal agar with determination of fluconazole sensitivity. Al Ameen J. Med. Sci., 6(2): 163-166.

33. Matan, N., H. Rimkeeree, A.J. Mawson, P. Chompreeda, V. Haruthaithanasan and M. Parker, 2006. Antimicrobial activity of cinnamon and clove oils under modified atmosphere conditions. Int. J. Food Microbiol., 107: 180-185.

34. Suhr, K.I., \& Nielsen, P.V. (2003). Antifungal activity of essential oils evaluated by two different application techniques against rye bread spoilage fungi. Journal of Applied Microbiology, 94(4), 665-674.

35. Radwan, I. \& Abed, Ahmed \& Abeer, Mohammad \& Ibrahim, M. \& Abdallah, Shamseldeen. (2014). Effect of Thyme, Clove and Cinnamon Essential Oils on Candida Albicans and Moulds Isolated from Different Sources. American Journal of Animal and Veterinary Sciences. 9. 303-314. 10.3844/ajavsp.2014.303.314. 